\title{
Gait Recognition with Fuzzy Classification Using Shoulder Body Joint
}

\author{
Jyoti Bharti and M. K. Gupta
}

\begin{abstract}
In this paper, the adopted methodology based on intersection with fuzzy classification. Consider two human body joint i.e. shoulder and feet. Feet body joint is separated in to two component toe and heel of the left and right leg. Two triangles are formed on joining the shoulder and feet (toe and heel). The intersecting points are computed on CASIA database. Fuzzy classification yield enhanced recognition rate.
\end{abstract}

Index Terms-Gait recognition, intersection, recognition rate, biometric, fuzzy.

\section{INTRODUCTION}

Biometrics is derived from Greek word "Bio" means life and "metrics" means measure[1].The term gait was first demonstrate by Johansson in 1970[2]. Some methods such as finger prints and face recognition, already proved to be very efficient in human recognition systems [3]. Face, fingerprint recognition, iris recognition can't work well from a large distances as well as on degraded images in present scenario security is one of the challenging issues in all field [1],[3]. Human gait is one of the promising biometric traits for identification. The gait is defined as "Gait is a particular way or manner of moving on foot". Human gait has a unique personal characteristic and cyclic in nature [3]. The main advantage of gait is that no human interference is required. It can work well even though the camera situated at large distance. In this paper gait recognition method is based at an intersecting points and compare fuzzy and nearest neighbor classifier to achieve better recognition rate.

This paper is an enhanced version of gait recognition with shoulder body joint [4].This paper is organized as follows. Section II described the existing method with their utility. Human gait and fuzzy concept described in section III. Section IV provides our proposed method. Experimental results \& analysis are presented in section $\mathrm{V}$, followed by Conclusions \& future scope in Section VI.

\section{RELATED WORK}

Many authors can worked on silhouettes images and Gait Energy images [5], [6], [7]. Haiping Lu et al. [8], [9] defined the body part 22 human parameter. The parameter defined the part length, width position and joint angles. C.

Manuscript received January 15, 2013; revised March 22, 2013.

Jyoti Bharti is with the Department of Comp.Sc and Engg. of Maulana Azad National Institute of Technology, Bhopal,India, (e-mail: jyoti2202@gmail.com)

M. K. Gupta is with the Electronics \& Communication Department of Maulana Azad National Institute of Technology, Bhopal, India (e-mail: gupta4@yahoo.com)
Senanyake et al. [10] used thigh, shank, foot, hip, ankle and knee body joint for normal gait sequences achieved better result on body joint. Faezeh Tafazzoli[11] extracted the gait motion of leg and arm. They computed the rotation variation of hip, knee and arm of silhouettes images and found better result with arm as compare to without arm variation. It is found from the related works, body joint plays an important role for identification and achieved better result on it.

\section{HUMAN GAIT AND FUZZY LOGIC}

\section{A. Human Gait}

Human identification through a style of walking Style of walking is called gait. Gait can be defined as "A peculiar way or manner one walks". Walking pattern can be analyzed by a gait cycle. Gait cycle is repeated motion of body part[6],[9]. The style of walking or gait cycle of every person is unique[5],[8]. Mostly there are no much more changes in head and shoulder motion as compare to hand and legs. The human gait cycle becomes when the initial position of foot becomes final position shown in Fig. 1. The single gait cycle is further divided into two phases:

Stance Phase: Foot touches the ground, loading response, Mid-stance, Terminal stance, and Pre-swing [5], [12].

Swing Phase: Foot does not contact with the ground. Initial swing, Middle swing, and Terminal swing [5], [12].

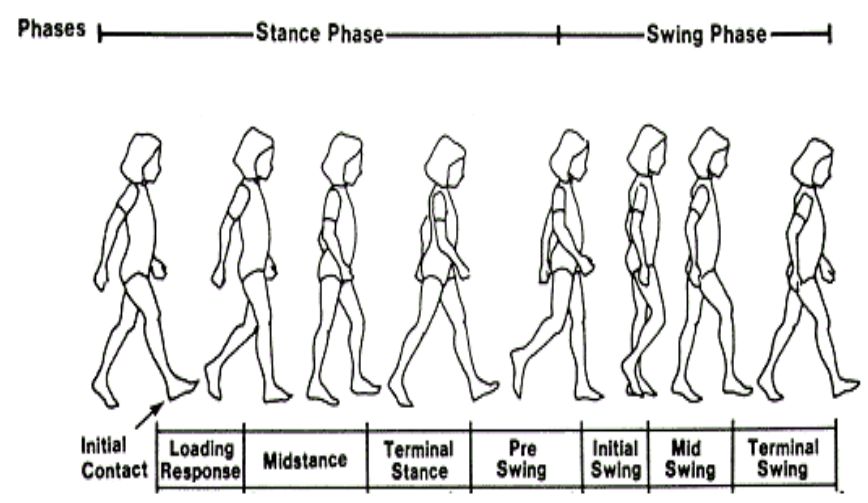

Fig. 1. Representation of subject moving through the gait cycle.

\section{B. Fuzzy Logic}

Fuzzy logic is mathematical approach to distinguish or classify the object based on the extracted feature values. In a classical way, an object takes on a value of either 0 or 1 . Fuzzy logic statements values are lies between $0 \& 1$. If the value is nearer to 0 then cannot recognize the person and value is closer to 1 then person is identified [13].

There are many types of membership function choose one of them dependent on the application. After testing and survey it decided that trapezoidal function is more suitable for our 
application. One of the most popular membership functions is trapezoidal function. It is defined by four parameters, $x \rightarrow$ $(a, b, c, d)$. A trapezoidal function formally described in (1) [12], [13].

\section{Representation of Membership Function}

There are many types of membership function choose one of them dependent on the application. After testing and survey it decided that trapezoidal function is more suitable for our application. One of the most popular membership functions is trapezoidal function. It is defined by four parameters, $x \rightarrow(a, b, c, d)$. A trapezoidal function formally described in (1) [12], [13].

$$
\mu A(x)=\left\{\begin{array}{c}
0, \text { for } x<a \\
\frac{x-a}{b-a}, \text { for } a \leq x \leq b \\
1, \text { for } b \leq x \leq c \\
\frac{d-x}{d-c}, c \leq x \leq d
\end{array}\right\}
$$

\section{Mamdani Fuzzy Models.}

Mamdani's fuzzy inference method is one the most commonly used fuzzy methodology. The output membership functions have to be fuzzy sets. After the aggregation process, there is a fuzzy set for each output variable that needs defuzzification. In most of the cases it is much more efficient [12], [13].

\section{Proposed Methodology}

In our proposed methodology concentrated on shoulder and feet body joints. Feet body joint component is subdivided into toe and heel of both left and right feet .Total five control or feature point are used and insert a white dot point on that control points shown in Fig. 2 [1], [4], [12].
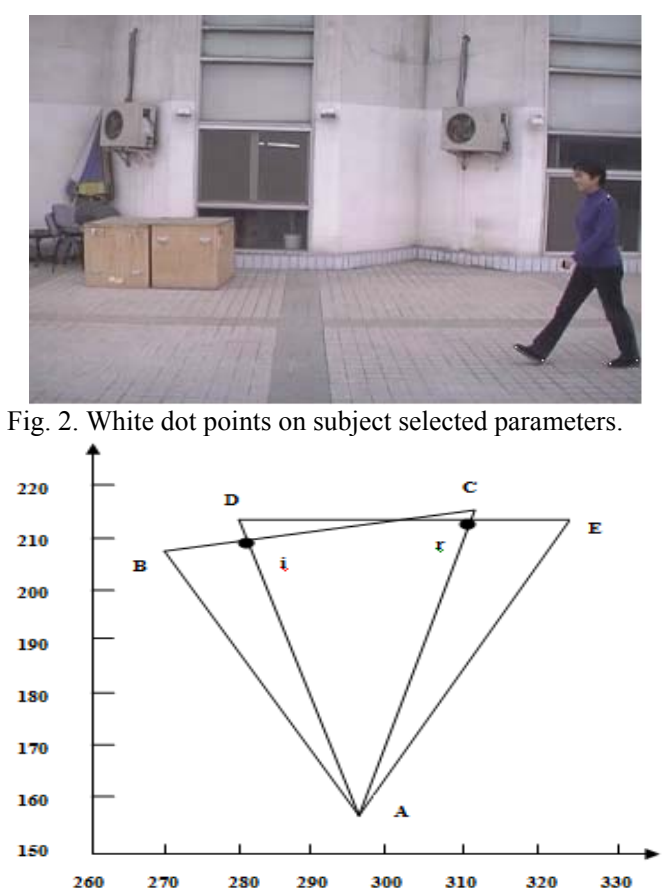

Fig. 3. I and I' are intersecting points between $\Delta$ 's ABC \& ADE.

After simulation these white dotted frames of each individual are simulated on MATLAB. Two triangles are formed on using these five featured point. First triangle is formed between shoulder and two heels of both feet (right heel and left heel) and similarly $2^{\text {nd }}$ triangle is formed between shoulder and two toe of both feet (right toe and left toe).These two triangle are intersect and two intersecting points are computed shown in Fig. 3

$I \& I$ are two intersecting points which have to be computed using parametric line equation. The extracted feature values are the Intersecting Points of the two Triangles. Triangle $\mathrm{ABC}$ and $\mathrm{ADE}$ are intersect at three places. But only two intersecting points $(i$ and $r$ ) are considered. Here point A represent shoulder and point B,C represents toe of both feet(left and right), point $\mathrm{D}, \mathrm{C}$ represents heel of both feet(Left and Right) shown in Fig. 3. Intersecting points are evaluated by solving the (2) \& (3).

$$
\begin{aligned}
& Y 1=\mathrm{m} 1 \cdot X 1+b 1 \\
& Y 2=\mathrm{m} 2 \cdot X 2+b 2
\end{aligned}
$$

Equalize the equation $2 \& 3$ but $\left(X_{1}=X_{2}\right)$

$$
\begin{gathered}
\mathrm{m} 1 . X 1+b 1=\mathrm{m} 2 . X 2+b 2 \\
X 1=\frac{(b 2-b 1)}{(\mathrm{m} 1-\mathrm{m} 2)}
\end{gathered}
$$

By solving (5) get the value of $x$ coordinate of point. Substitute $X_{1}$ value in (2) then $y$ coordinate point is computed. The coordinate of point $\mathrm{i}$ is $\left(X_{1}, Y_{1}\right)$.

Similar process is used for finding the others Intersecting Point $r$ of Line DE and AC. The coordinate of point $r$ is $(X r$, Yr) .

So we get the Intersecting Points $i\left(X_{i}, Y_{i}\right)$ and $\mathrm{r}\left(X_{r}, Y r\right)$.

After getting the intersecting point, the two mean values of an intersecting point of an individual subject are computed shown in (6).

$$
\bar{x}=\frac{1}{n} \sum_{i=1}^{n} x_{i}
$$

After computation of mean intersecting values of two triangle. These mean intersecting value are input of fuzzy inference system(FIS). FIS compare and produces result with the database values. Their results are based on the following fuzzy rules:

1) If (I1 is yes) and (I2 is yes) and (I3 is yes) and (I4 is yes) then (Match is Excellent)

2) If (I1 is No) and (I2 is yes) and (I3 is yes) and (I4 is yes) then (Match is Good)

3) If (I1 is yes) and (I2 is No) and (I3 is yes) and (I4 is yes) then (Match is Good)

4) If (I1 is yes) and (I2 is yes) and (I3 is No) and (I4 is yes) then (Match is Good)

5) If (I1 is yes) and (I2 is yes) and (I3 is yes) and (I4 is No) then (Match is Good)

6) If (I1 is No) and (I2 is No) and (I3 is yes) and (I4 is yes) then (Match is Average)

7) If (I1 is yes) and (I2 is No) and (I3 is No) and (I4 is yes) then (Match is Average)

8) If (I1 is yes) and (I2 is yes) and (I3 is No) and (I4 is No) then (Match is Average)

9) F (I1 is No) and (I2 is yes) and (I3 is yes) and (I4 is No) then (Match is Average) 
10) If (I1 is No) and (I2 is yes) and (I3 is No) and (I4 is Yes) then (Match is Average)

11) If (I1 is Yes) and (I2 is No) and (I3 is yes) and (I4 is No) then (Match is Average)

12) If (I1 is yes) and (I2 is No) and (I3 is No) and (I4 is No) then (Match is Poor)

13) If (I1 is No) and (I2 is yes) and (I3 is No) and (I4 is No) then (Match is Poor)

14) If (I1 is No) and (I2 is No) and (I3 is yes) and (I4 is No) then (Match is Poor)

15) If ( $\mathrm{I} 1$ is No) and (I2 is No) and (I3 is No) and (I4 is yes) then (Match is Poor)

16) If (I1 is No) and (I2 is No) and (I3 is No) and (I4 is No) then (Match is No match)

\section{A. Proposed Algorithm}

1) Select input subject frames are(17,20,23,25 and 27).

2) Convert color image into gray scale image.

3) Compute pixel values of white dotted point on body joint.

4) Two triangle are constructed.

5) Calculate two intersection point for these triangle.

6) Mean values are computed.

7) These mean values are used for recognition by fuzzy classifier.

8) The mean values is compared with the database. If the mean values is found then the person is guaranteed to found. It not person not found.

9) Repeat the process for another user.

\section{EXPERIMENTAL RESULT AND ANALYSIS}

TABLE I: RR FOR INTERSECTION FUZZY -SHOULDER

\begin{tabular}{|l|l|l|l|l|l|l|}
\hline \multicolumn{7}{|c|}{ Shoulder Fuzzy - Intersection } \\
\hline Frames & Rank & Side1 & Side2 & \multicolumn{2}{|l|}{ Side(S1+S2) } & $\begin{array}{l}\text { Average } \\
(\%)\end{array}$ \\
\hline & & & & S1 & S2 & \\
\hline 23 & $R 1$ & $20 \%$ & $0 \%$ & $0 \%$ & $10 \%$ & $7.50 \%$ \\
\hline & $R 5$ & $50 \%$ & $10 \%$ & $40 \%$ & $20 \%$ & $30.00 \%$ \\
\hline 20 & $R 1$ & $20 \%$ & $10 \%$ & $10 \%$ & $10 \%$ & $12.50 \%$ \\
\hline & $R 5$ & $60 \%$ & $50 \%$ & $60 \%$ & $30 \%$ & $50.00 \%$ \\
\hline 17 & $R 1$ & $100 \%$ & $70 \%$ & $90 \%$ & $70 \%$ & $82.50 \%$ \\
\hline & $R 5$ & $100 \%$ & $100 \%$ & $100 \%$ & $100 \%$ & $100.00 \%$ \\
\hline 25 & $R 1$ & $30 \%$ & $10 \%$ & $30 \%$ & $0 \%$ & $17.50 \%$ \\
\hline & $R 5$ & $100 \%$ & $70 \%$ & $100 \%$ & $70 \%$ & $85.00 \%$ \\
\hline 27 & $R 1$ & $90 \%$ & $100 \%$ & $100 \%$ & $100 \%$ & $97.50 \%$ \\
\hline & $R 5$ & $100 \%$ & $100 \%$ & $100 \%$ & $100 \%$ & $100.00 \%$ \\
\hline
\end{tabular}

In proposed work, ten user have been taken from Institute of Automation of Chinese Academy of Sciences (CASIA) data [14] with two sides view $s 1 \& s 2$. S1 sides person is walking from right to left. While $\mathrm{s} 2$ side, person is walking from left to right. Database is made up of 23 frames for all sides $s 1, s 2 \&(s 1+s 2)$ data contain both the values of $s 1 \&$ $s 2$ sides. For testing, input frames are taken except 23 frames of data. Randomly select as an input frames with the number of frames are, 17, 20, 23, $25 \& 27$ are used. 23 number of frames are expected to be a complete gait cycle. While other are incomplete and over gait cycle. Proposed work is simulated on MATLAB. After simulation the result are evaluated in the form of Rank1 (R1) \& Rank5 (R5). Rank1 $(R 1)$ is a exact matching while Rank5 $(R 5)$ is a top 5 matches with the database. Intersecting points are evaluated for each input types frames. Evaluated intersecting values are used as a input values in FIS system. The FIS system produces decision based on the 16 rules. The evaluated results are shown in Table I.

The Fig. 4 and Fig. 5 represent the graphical representation of Table I.

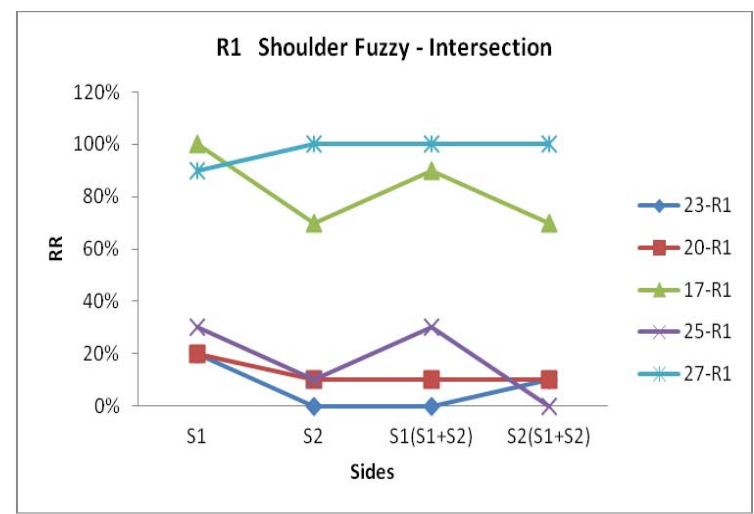

Fig. 4. RR v/s sides for shoulder fuzzy for R1

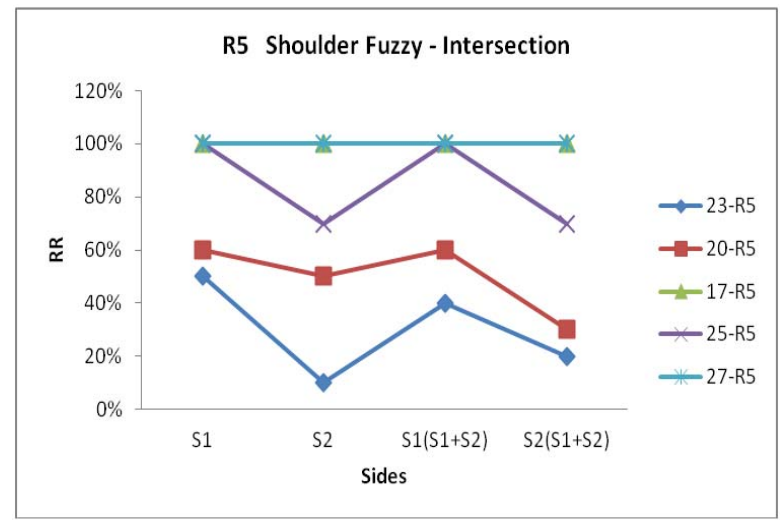

Fig. 5. RR v/s sides for shoulder fuzzy for R5

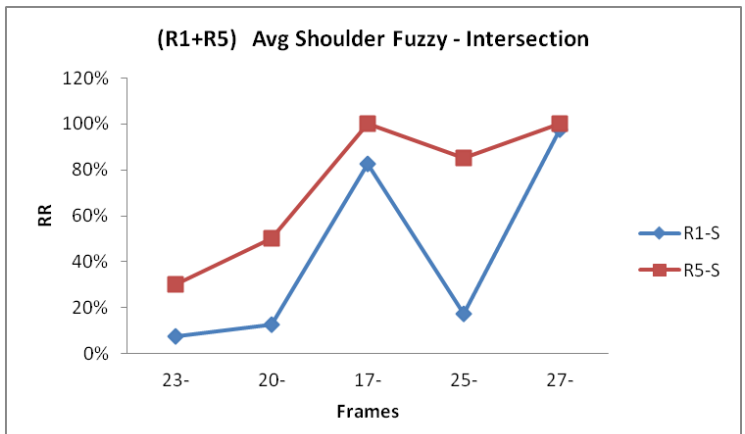

Fig. 6. RR v/s frames for shoulder fuzzy for AVG R1\& AVG R5

From Fig. 4 RR for 27 is best for all sides (100\%), except at $S 1(90 \%)$ and for 17 frames it is satisfactory. At this 17 frames RR at $S 2$ sides is less as compared to $S 1$ Sides. RR become $70 \%$ at $\mathrm{S} 2$ (Ind and $S 1+S 2$ ) but at $S 1, \mathrm{RR}$ is 90 $100 \%$. RR of 23,20 and 25 frames is below $40 \%$ for all sides.

From Fig. 5 At 17 and 27 frames RR is best (100\%) for all sides. RR of 25 frames is also best (100\%) for S1 sides and $\operatorname{good}(70 \%$ for $\mathrm{S} 2$ sides). RR for 20 is satisfactory except at $\mathrm{S} 2(\mathrm{~S} 1+\mathrm{S} 2)$. For 23 frames RR is not good.

As per Fig. 6 is a graphical comparison of R1 and R5 average fuzzy shoulder corresponds to table 1 . It is found that $R 5$ produce better result to $R 1$. 
Fig. 7 and Fig. 8 is a comparision of fuzzy $\&$ without fuzzy (nearest neighbour classification) [12].

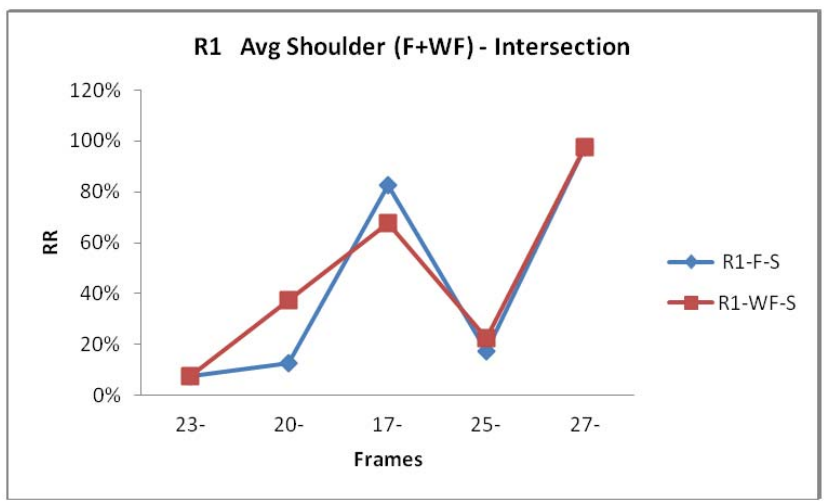

Fig. 7. RR v/s Frames for Shoulder for fuzzy \& without Fuzzy for AVG R1.

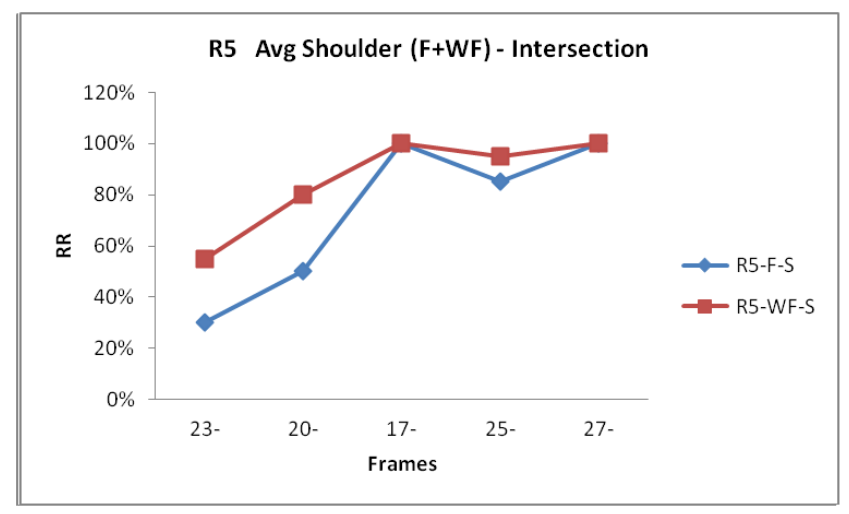

Fig. 8. RR v/s frames for shoulder fuzzy \& without fuzzy for AVG R5.

TABLE II: BEST FRAME FOR FUZZY AND WiTHOUT FUZZY.

\begin{tabular}{|c|c|c|c|c|}
\hline \multicolumn{5}{|c|}{ Intersection( Shoulder Body Joint) } \\
\hline & \multicolumn{5}{|c|}{ Fuzzy } \\
\hline & R1 & RR & MAX & Sides \\
\hline S & 27 & $97.5 \%$ & $100 \%$ & All Sides \\
\hline S & 17 & $82.5 \%$ & $100 \%$ & S1 Side \\
\hline & R5 & RR & MAX & Sides \\
\hline S & 27 & 100 & $100 \%$ & All Sides \\
\hline S & 17 & 100 & $100 \%$ & All Sides \\
\hline \multicolumn{5}{|c|}{ Without Fuzzy[4] } \\
\hline & R1 & RR & MAX & Sides \\
\hline S & 27 & 97.5 & $100 \%$ & All Sides \\
\hline & R5 & RR & MAX & Sides \\
\hline S & $17 \& 27$ & 100 & $100 \%$ & All Sides \\
\hline
\end{tabular}

As per Fig. 7 Fuzzy produces better RR (82.5\% and 97.5\%) for 17 and 27 frames. At 25 frames RR is average $(20 \%)$ and for 23 and 20 frames $R R$ is very poor $(<=25 \%)$. We achieve best RR (100\%) for without fuzzy at 27 frames and good RR (67.5\%) at 17 frames. Performances of fuzzy and without fuzzy are almost same at 27 frames. But at 17 frame fuzzy is better than without fuzzy.

As per Fig. 8 at 17, 25 and 27 frames fuzzy and without fuzzy both produces almost similar and best RR (approximately $100 \%$ ) but at 23 ad 20 frames RR of without fuzzy is good (55-80\%) and average RR for fuzzy (30-50\%). RR of fuzzy is better.

As per the Fig. $7 \& 8$ and table II, it is concluded that fuzzy produce better RR at $27 \& 17$ frame while without fuzzy produce better on 27 frame only. Fuzzy classification is better to nearest neighbor classifier for R1 but for R5 without fuzzy is better.

\section{CONCLUSION}

It is concluded that the featured point form unique intersection point for individuals. For R1 fuzzy has enhanced RR than without fuzzy. Without fuzzy is better than fuzzy for R5 at all frames.

\section{FUTURE WORK}

In present work shoulder body joint is consider with fuzzy classification. Use other classification method like SVM, Neuro Fuzzy etc than compare and find that which one is better.

\section{CONCLUSION}

A conclusion section is not required. Although a conclusion may review the main points of the paper, do not replicate the abstract as the conclusion. A conclusion might elaborate on the importance of the work or suggest applications and extensions.

\section{REFERENCES}

[1] M. P. Murray, "Gait as a total pattern of movement,"American Journal of Physical Medicine, vol. 46, no. 1, pp. 290-333, 1967.

[2] G. Johnsson, "Visual perception of biological motion and a model for its Analysis, Perception Psychophys," in Proc. IEEE Conference on Intelligent Control and Automation, vol. 14, no. 2, pp. 201-211, 1973.

[3] J. P. Singh and S. Jain, "Person Identification Based on Gait using Dynamic Body Parameters," in Proc. IEEE International Conference on Trendz in Information Sciences \& Computing (TISC), pp. 248-252, 2010.

[4] J. Bharti and M. K. Gupta, "Gait Recognition using shoulder body joint," International Journal of Computer Applications, vol. 53, no. 4, pp. 49-52, September 2012.

[5] J. R. Gage, P. A. Deluca, and T. S. Renshaw, "Gait analysis principles and applications," The Journal of Bone and Joint Surgery, vol.77, pp. 1607-1623, 1995.

[6] N. V. Boulgouris, D. Hatzinakos, and K. N. Plataniotis, "A challenging signal processing technology for biometric identification," IEEE Signal Processing Magazine, vol. 22, no. 6, pp. 78-90, November 2005.

[7] J. P. Ferreira, M. Crisostomo, A. P. Coimbra, D. Carnide, and A. Marto, "A Human Gait Analyzer," in Proc. IEEE International Symposium on Intelligent Signal Processing, pp.1-5 October 2007.

[8] H. P. Lu, K. N. Plataniotis, and A. N. Venetsanopoulos, "A Full-body layered deformable model for automatic model-based gait recognition," Journal on Advances in Signal Processing EURASIP, vol. 2008, no. 13, Jan 2008.

[9] H. P. Lu, K. N. Plataniotis, and A. N. Venetsanopoulos, "Layered deformable model for gait analysis," in Proc. IEEE seventh International Conference on Automatic Face and Gesture Recognition ( $\left.F G^{\prime} 06\right)$, pp. 249-256, 2006.

[10] C. Senanayake and S. M. N. A. Senanayake, "Fuzzy logic based implementation of a real-time gait phase detection algorithm using kinematical parameters for walking," in Proc. IEEE International Conference of Soft Computing and Pattern Recognition (SOCPAR'09), pp. 586-591, 2009.

[11] F. Tafazzoli and R. Safabakhsh, "Model-based human gait recognition using leg and arm movements," Elsevier Journal Engineering Applications of Artificial Intelligence, vol. 23, no. 8, pp. 1237-1246, Dec. 2010.

[12] J. Bharti and M. K. Gupta, "Gait recognition with geometric characteristic and fuzzy logic," Canadian Journal on Image Processing and Computer Vision, vol. 3, no. 1, pp. 6-11, March 2012.

[13] V. K. Lamba, Neuro Fuzzy Systems, First Edition, 2008, pp. 22-24, 47-52,58,79,85.

[14] Center for Biometrics and Security Research (CBSR). 


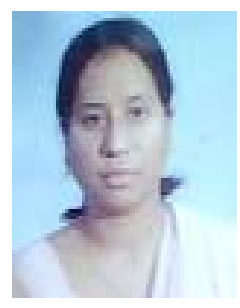

Jyoti Bharti was born in 1976, obtained Bachelor of Engineering degree in Computer Science and Engineering in 2000 from Lakshmi Narain College of Technology affiliated to Barkatullah University Bhopal. Did Masters of Technology from Samrat Ashok Technical Institute Vidisha affiliated to Rajiv Gandhi Proudyogiki Vishwavidyalaya Bhopal, MP, India. Research area is Image processing and Data mining. Total teaching

Experience is more than 10 years. After serving 3 years in Lakshm Narain College of Technology and RKDF Institute of Science and Technology Bhopal, MP, India, joined the Department of Information Technology, Maulana Azad National Institute of Technology, and Bhopal as lecturer. Currently serving the Institute as Assistant Professor in Department of Information Technology. Persuing the PhD work in the area of Image processing. Published more than 10 papers in various National International conferences, journals and has attended more than 15 short term courses on Image processing, Data mining, and Security

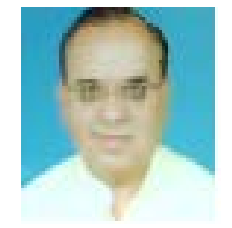

Mahendra Kumar Gupta was born in 1953, obtained Bachelor of Engineering degree in Electronics Engineering in 1975 from Maulana Azad National Institute of Technology Bhopal, MP, India (formerly known as Maulana Azad College of Technology, Bhopal), .Did Master of Engineering in control and Guidance from Dept of Electronics and Communication Engineering, Indian institute of Technology Roorkee (Formerly Known as University of Roorkee in 1992), Uttrakhand, India. Obtain the doctor of philosophy (PhD) from Barkatullah University Bhopal, MP, and India in 2006. His area of research is VLSI application to Control Engineering

Initially after serving more than 5 years in industries, He joined Department of Electronics \& Communication of Maulana Azad National Institute of Technology Bhopal, MP, India (formerly known as Maulana Azad College of Technology) as lecturer in 1985 and currently he is serving the Institution as Professor in Department Electronics \& Communication. He has published 15 Papers in reputed National $\backslash$ International Journal and presented more than 10 papers in various national \International Conferences. His area of interest is Microprocessor, Image Processing, Integrated Circuits, Control and VLSI.

Dr. Gupta is member IEEE (to be renewed), Life Member IE (I), Fellow member IETE, Life Member CSI and Life Member ISTE. 